\title{
The problem with the non-idealness of the MHD heliosheath
}

\author{
V. B. Baranov ${ }^{1}$ and H.-J. Fahr ${ }^{2}$ \\ ${ }^{1}$ Institute for Problems in Mechanics, Russian Academy of Sciences, 101-1 ,Vernadskii Ave., Moscow, 119526, Russia \\ ${ }^{2}$ Inst. for Astrophysics and Space Research, University of Bonn, Auf dem Hügel 71, D-53121 Bonn, Germany
}

Received: 9 November 2005 - Revised: 15 February 2006 - Accepted: 10 May 2006 - Published: 15 June 2006

\begin{abstract}
When describing the plasma - field behaviour in the heliospheric interface the difficulty arises that classical MHD concepts are not fully applicable to this complicated multifluid interaction scenario. The classical MHD concept of ideally frozen-in magnetic fields is only strictly valid, if the magnetized medium is fully ionized. As is well known, however, the heliospheric medium represents a partially ionized plasma which contains neutral $\mathrm{H}$-atom flows interacting with the ions via resonant charge exchange processes. Caused by this cross-interaction between the neutral and the ionized media additional non-classical currents are driven which induce additional magnetic fields. These latter fields can be shown to show the tendency to diffuse relative to the ion bulk motion. As we can show this non-classical diffusion is especially pronounced near plasma boundaries or shocks and there act in a way to dissolve the abruptness in the transition structure of the plasma properties. Here we give first estimates of these effects and point to a need to revise the classical MHD theory at its application to partially ionized media.
\end{abstract}

\section{Introduction}

As is well known since long (Baranov et al., 1970) the interaction of supersonic flow of the local interstellar plasma component (electrons and protons) with the supersonic solar wind gives rise to the formation of an outer bow shock, a tangential discontinuity (called heliopause) and an inner termination shock (see BS, HP and TS, respectively, in Fig. 1). The region between BS and TS (the so-called interface) is separated by the heliopause into two regions: the outer heliosheath (decelerated and subsonic flow of the interstellar plasma component between BS and HP) and the inner heliosheath (decelerated and subsonic solar wind between TS

Correspondence to: H.-J. Fahr (hfahr@astro.uni-bonn.de)

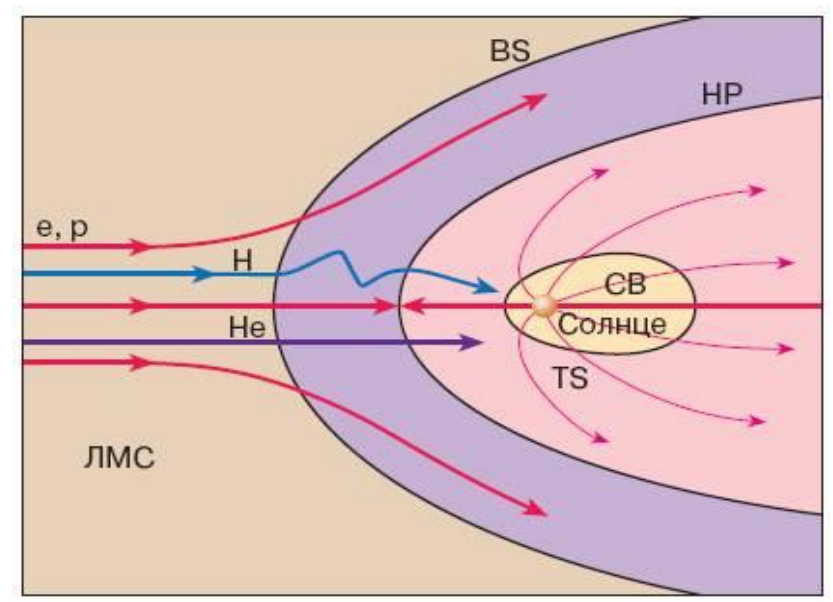

Fig. 1. Qualitative picture of the solar wind interaction with the supersonic flow of the local interstellar medium (LISM). Here BS is the bow shock, HP is the heliopause, TS is the solar wind termination shock. The region between the BS and HP is called the outer heliosheath, the one between TS and HP is called the inner heliosheath.

and HP). In detailed studies of this interaction scenario, however, the important fact has to be taken into account that the local interstellar gas is a partially ionized plasma. Respecting this fact the interstellar $\mathrm{H}$-atom flow, in addition to the fully ionized plasma flow, needs a separate description. As it turns out, this neutral flow cannot adequately be described in the framework of a hydrodynamic approximation, because the mean free path of $\mathrm{H}$-atoms with respect to the dominant process, i.e. resonant charge exchange with protons, is comparable with the characteristic length of the problem (for example, with the extent of the heliosheath $\sim 50 \mathrm{AU}$ ), i.e. the $\mathrm{H}$-atom distribution function, due to the essentially collisionless situation, cannot be taken as a shifted Maxwellian. 
It is evident from classical literature that shocks and tangential discontinuities can only be formed in the framework of the ideal Euler hydrodynamics, where second order derivatives are absent. Below we will consider a possible effect of the interstellar magnetic field on the outer heliosheath structure taking into account the interstellar plasma component as well as the neutral $\mathrm{H}$-atom flow. The magnetohydrodynamic (MHD) eqnarrays to describe the partially ionized flow around the heliopause (see, for example, Florinskii et al., 2003) are critically analysed in Sect. 2. It is shown below that under the actually prevailing conditions in the framework of the classic MHD (Kulikovskii and Lyubimov, 1965) a diffusion of the interstellar magnetic field in the outer heliosheath can become important due to ion currents (Baranov and Fahr, $2003 \mathrm{a}, \mathrm{b}$ ). It seems to us that the earlier results of a kinetic-MHD approach (Baranov and Malama, 1993), again used for the numerical solution of the above mentioned problem (Izmodenov et al., 2005), confirm the earlier assertion by Baranov and Fahr (2003a,b). These latter results will be shown in Sect. 3.

\section{MHD-formulation of the problem}

To solve the problem of the interaction between the supersonic flow of the interstellar gas and the solar wind in the presence of an interstellar magnetic field in several cases in the literature a multifluid model has been used (see, for example, Florinskii et al., 2003; Fahr et al., 2000), although the Knudsen number for H-atoms $K n=l / L \sim 1$ ( $l$ is mean free path of H-atoms with respect to resonant charge exchange with protons, $L$ is the characteristic length of the interaction region, for example, the extent of the heliosheath). In this case the momentum conservation eqnarrays for a partially ionized hydrogen gas in the presence of an interstellar magnetic field have the form (stationary problem!):

$$
\begin{aligned}
\left(\mathbf{V}_{\alpha} \cdot \nabla\right) \mathbf{V}_{\alpha}=-\frac{1}{m_{\alpha} n_{\alpha}} \nabla & p_{\alpha}+e_{\alpha}\left(\mathbf{E}+\frac{1}{c} \mathbf{V}_{\alpha} \times \mathbf{B}\right) \\
& +\sum \mathbf{R}_{\alpha \beta}, \mathbf{R}_{\alpha \beta}=\frac{\mathbf{V}_{\alpha}-\mathbf{V}_{\beta}}{\tau_{\alpha \beta}}
\end{aligned}
$$

where the indices $\alpha=e, p, H$ are representative for electrons, protons and H-atoms, $\mathbf{V}, \mathbf{E}, \mathbf{B}$ and $\mathbf{R}$ are vectors of the bulk velocity, the electric field, the magnetic field induction and the mutual interaction force due to exchange of momentum between components $\alpha$ and $\beta$ due to mutual collisions, respectively, $m, n, p$ and $\tau$ are denote the mass of the particle, the number density, the static pressure and the mean free-flight time. The particle flux continuity eqnarray has the form

$$
\nabla \cdot n_{\alpha} \mathbf{V}_{\alpha}=0
$$

and the eqnarray of the magnetic field induction can be expressed in a form which determines magnetic field freezing in the proton flow (Florinskii and Zank, 2003; Baranov and Fahr, 2003b)

$\nabla \times\left(\mathbf{V}_{p} \times \mathbf{B}\right)=0$.

To complete and close the system of eqnarrays one must add the eqnarrays of state and of energy conservation for all components as well as Maxwell eqnarrays for the electromagnetic fields.

On the basis of the above closed system of multifluid eqnarrays we could obtain the eqnarrays of the classic one-fluid MHD theory (see, for example, Kulikovskii and Lyubimov, 1962) by simple manipulations and redefinitions. Namely the momentum and continuum eqnarrays take the form

$(\mathbf{V} \cdot \nabla) \mathbf{V}=-\frac{1}{\rho} \nabla p+\frac{1}{c} \mathbf{j} \times \mathbf{B} ; \nabla \cdot \rho \mathbf{V}=0$,

where $\rho \mathbf{V}=\sum m_{\alpha} n_{\alpha} \mathbf{V}_{\alpha}, \rho=\sum m_{\alpha} n_{\alpha}, p=\sum p_{\alpha}$ determine the bulk velocity $\mathbf{V}$, the mass density $\rho$ and the static pressure $p$ of the fluid consisting of electrons, protons $\left.\left(n_{e}\right], \approx n_{p}\right)$ and $\mathrm{H}$-atoms. The electric current is determined by the formula

$\mathbf{j}=-e n_{e}\left(\mathbf{V}_{e}-\mathbf{V}_{p}\right)$,

where $|e|$ is the absolute proton or electron charge. Manipulations with the momentum Eq. (1) give rise to the generalized Ohm's law for a partially ionized plasma in the form (Kulikovskii and Lyubimov, 1965; Cowling, 1976; Baranov and Fahr, 2003a)

$$
\begin{aligned}
\mathbf{j}=\sigma\left(\mathbf{E}+\frac{1}{c} \mathbf{V}\right. & \times \mathbf{B})+\frac{e \tau_{e p}}{m_{e}}\left(\nabla p_{e}-\frac{1}{c} \mathbf{j} \times \mathbf{B}\right) \\
& +\frac{\Lambda}{B^{2}}\left[\frac{s}{1-s} \nabla p \times \mathbf{B}+\frac{1}{c}(\mathbf{j} \times \mathbf{B}) \times \mathbf{B}\right],
\end{aligned}
$$

where $\Lambda=c(1-s)^{2} \omega_{e} \tau_{e p} \omega_{p} \tau_{p H}, \quad s=n_{e} /\left(n_{e}+n_{H}\right)$ and $\sigma=n_{e} e^{2} \tau_{e p} / m_{e}$ are the ionization degree and the electric conductivity, respectively, $\omega_{\alpha}(\alpha=e, p)$ is the gyrofrequency of charged particles in the presence of the magnetic field, i.e. Larmor frequency. It should be noted here, that in the outer heliosheath (see Fig. 1) one finds $\omega_{\alpha} \tau_{\alpha \beta} \gg 1$.

As was shown by Baranov and Fahr (2003) the last term of Eq. (6) is comparable or larger than the term $\sigma / c(\mathbf{V} \times \mathbf{B})$ for the problem considered, i.e. the Eq. (6) can be re-written in the following form

$$
\begin{aligned}
\mathbf{E}= & -\frac{1}{c}(\mathbf{V} \times \mathbf{B})- \\
& -\frac{\Lambda}{B^{2} \sigma}\left[\frac{s}{1-s} \nabla p \times \mathbf{B}+\frac{1}{c}(\mathbf{j} \times \mathbf{B}) \times \mathbf{B}\right]
\end{aligned}
$$

at $\omega_{e} \tau_{e p} / \operatorname{Re}_{m} \ll 1$, where $\operatorname{Re}_{m}=4 \pi \sigma V_{\infty} L / c^{2} \gg 1$, where $\mathrm{Re}_{m}$ is the magnetic Reynolds number. The last inequality due to the finite electric conductivity can be proven to be fulfilled in the region of the heliosheath considered here. In this case the Hall current and the magnetic field diffusion due to 
the finite electric conductivity can be neglected. Equation (7) leads to the eqnarray of the magnetic field induction (for the stationary problem, i.e. $\nabla \times \mathbf{E}=0$ ) in the form

$$
\begin{aligned}
\nabla & \times(\mathbf{V} \times \mathbf{B})=-\nabla \\
& \left.\times\left\{\frac{c \Lambda}{B^{2} \sigma}\left[\frac{s}{1-s} \nabla p \times \mathbf{B}+\frac{1}{4 \pi}[(\nabla \times \mathbf{B}) \times \mathbf{B})\right] \times \mathbf{B}\right]\right\}
\end{aligned}
$$

The right hand side (RHS) of this equation is determined by the proton current $\mathbf{j}_{p}$ (see, for example, Baranov and Fahr, $2003 a, b)$ which is given by

$\mathbf{j}_{p}=\frac{2 e \tau_{p a}(1-s)^{2}}{m_{p}}\left(-\frac{s}{1-s} \nabla p+\frac{1}{c} \mathbf{j} \times \mathbf{B}\right)$.

We see from Eq. (8), that the magnetic field is not frozen in into the plasma. To determine the coefficient of a differential diffusion let us consider the RHS last term of Eq. (8). We obtain, when using some vector identities, the following equalities

$$
\begin{aligned}
{[(\nabla \times \mathbf{B}) \times \mathbf{B}] \times \mathbf{B} } & =-\mathbf{B} \times[(\nabla \times \mathbf{B}) \times \mathbf{B}] \\
& =\left[-B^{2} \nabla \times \mathbf{B}+\mathbf{B}(\mathbf{B} \cdot \nabla \times \mathbf{B})\right] .
\end{aligned}
$$

First we take into account only the first term on the RHS of this equality and obtain

$\nabla \times\left(B^{2} \nabla \times \mathbf{B}\right)=-B^{2} \nabla^{2} \mathbf{B}+\nabla \times B^{2}(\nabla \times \mathbf{B})$,

where the first term of the RHS determines the diffusion of the magnetic field. Our estimations then show (Baranov and Fahr, 2003a) that

$$
|\nabla \times(\mathbf{V} \times \mathbf{B})| \leq \kappa\left|\nabla^{2} \mathbf{B}\right|
$$

where the diffusion coefficient $\kappa$ due to the proton current $\mathbf{j}_{p}$ is determined by the formula (see Eq. (8))

$\kappa=\frac{c \Lambda}{4 \pi \sigma} \quad\left[\frac{c m^{2}}{s}\right]$.

As is evident, we retain the ideal magnetic field freezing in case of the fully ionized plasma, namely at $s=1$. In the framework of the multifluid MHD, where $K n<1$, and at $s \neq 1$, we have $\kappa / V L \geq 1$, with $L=50 \mathrm{AU}$ being the scale of the heliosheath for the problem of the outer heliosheath structure, i.e. the diffusion of the interstellar magnetic field becomes important. In this case the bow shock is absent due to the presence of the second derivatives in the system of MHD eqnarrays. This result cannot be depending on the use of a multi-fluid or one-fluid approximation, because the transition from first to second order approximation is a result of identical transformations.

\section{Mathematical formulation of a kinetic-MHD flow in the outer heliosheath and first results of calculations.}

The solution of the MHD structure of the outer heliosheath will be satisfactorily correct, if in view of $K n \sim 1$ one uses a kinetic- MHD approximation to describe the $\mathrm{H}$-atom flow. The MHD eqnarrays for the plasma components (only electrons and protons) have the form

$\nabla \cdot \rho \mathbf{V}=0$,

$(\mathbf{V} \cdot \nabla) \mathbf{V}+\frac{1}{\rho} \nabla p=\frac{1}{4 \pi \rho}(\nabla \times \mathbf{B}) \times \mathbf{B}+\mathbf{F}_{1}$,

$\nabla \cdot \rho \mathbf{V}\left(\frac{V^{2}}{2}+\frac{\gamma}{\gamma+1} \frac{p}{\rho}\right)=F_{2}$,

$\nabla \times(\mathbf{V} \times \mathbf{B})=0$,

$\nabla \cdot \mathbf{B}=0$,

$\left(\mathbf{V} \approx \mathbf{V}_{p}, \rho \approx m_{p} n_{p}\right)$

Here the functions $\mathbf{F}_{1}\left(f_{H}\right)$ and $F_{2}\left(f_{H}\right)$ determine the rate of momentum and energy change of the plasma component due to charge exchange interaction processes of $\mathrm{H}$-atoms with protons.

The already developed Monte-Carlo method (Baranov and Malama, 1993) to obtain the solution of the coupled nonlinear Boltzmann eqnarray for the $\mathrm{H}$-atom distribution function $f_{H}$ (the distribution function of the protons $f_{p}$ is assumed to be a shifted Maxwellian) is now used to determine the above introduced functions $\mathbf{F}_{1}$ and $F_{2}$.

The axis-symmetric 2D problem of the solar wind interaction with the partially ionized interstellar plasma in the presence of the interstellar magnetic field was solved by Alexashov et al. (2000) at $\mathbf{B} \| \mathbf{V}$, and more recently the associated 3D- problem was calculated by Izmodenov et al. (2005). These authors kindly calculated for us the enlightening unpublished results shown in Figs. 2 through 4. In these Figures the distributions of temperature, electron (proton) number density, and flow lines are presented. To obtain these results it was assumed that the number density, the bulk velocity and the temperature of the solar wind at the Earth's orbit are equal to: $n_{p, E}=n_{e, E}=7.39 \mathrm{~cm}^{-3}, V_{E}=432 \mathrm{~km} \mathrm{~s}^{-1}, T_{E}=51109 \mathrm{~K}$, respectively. The corresponding parameter values for the local intercloud (LIC) medium are accepted to be equal to: $n_{p, \mathrm{LIC}}=n_{e, \mathrm{LIC}}=0.06 \mathrm{~cm}^{-3}, V_{\mathrm{LIC}}=26.4 \mathrm{~km} \mathrm{~s}^{-1}$, $T_{\mathrm{LIC}}=6527 \mathrm{~K}$. The angle $\theta$ between the interstellar magnetic field $\mathbf{B}_{\text {LIC }}$ and the vector of the bulk velocity $\mathbf{V}_{\text {LIC }}$ at Figs. 2 through 4 is equal to $\theta=\pi / 4,\left|\mathbf{B}_{\mathrm{LIC}}\right|=2.5 \mu \mathrm{G}$ and the ratio of solar radiation to gravitation forces is taken to be $\mu=1$.

Inspection of Fig. 2 and Fig. 3 tells us that there are abrupt jumps of plasma temperature and number density in the case of $\mathbf{B}=0$ (dotted lines), i.e. a typical bow shock is formed, if the interstellar magnetic field is absent. In contrast, solid lines are drawn for the case $\mathbf{B} \neq 0$ revealing the fact that in presence of a finite interstellar magnetic field one has to expect a smooth transition of the plasma parameters, i.e. the typical bow shock is dissolved. The last claim is also confirmed by smooth flow lines presented in Fig. 4. We think that results presented in Fig. 2 through Fig. 4, calculated on the basis of this above mentioned magnetohydrokinetic 


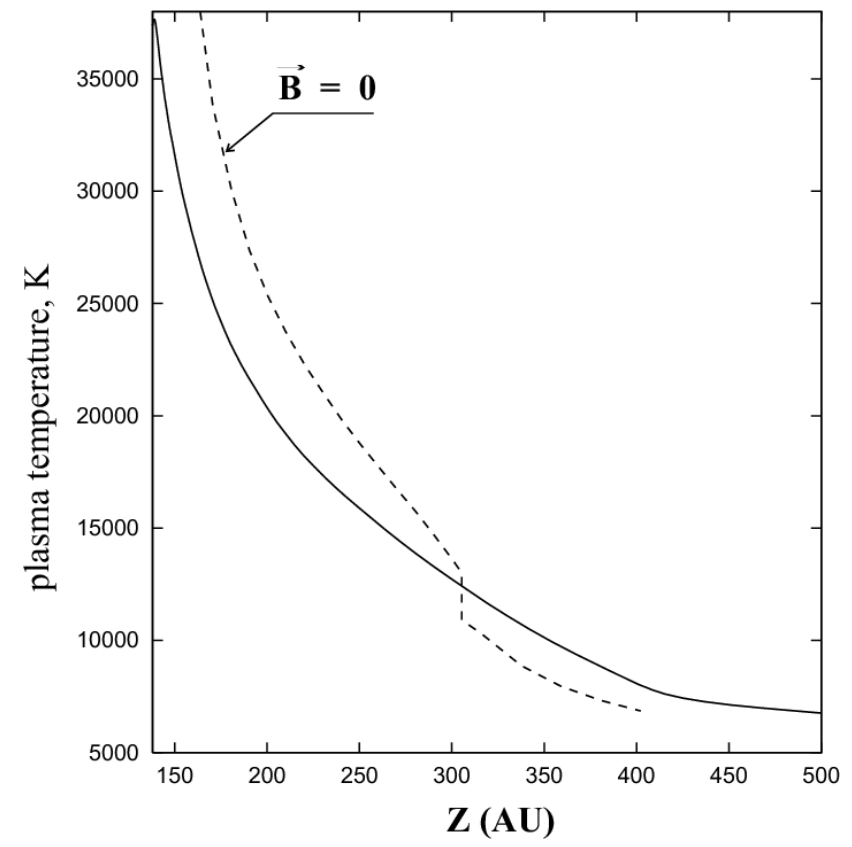

Fig. 2. Solutions for the plasma temperature as function of the solar distance are shown on the upwind axis: Dashed lines are obtained for vanishing interstellar magnetic field (i.e. $\mathrm{B}=0$ ), fully drawn lines are valid for a finite magnetic field $B=2.5 \mu G \geq 0$.

MHD approximation, confirm our conclusions already obtained in Sect. 2 on the basis of a pure MHD approximation (the bow shock cannot be formed as solution of a system of MHD eqnarrays which keep the second order derivatives).

\section{Kinetically enforced deviations from frozen-in flows}

As demonstrated above it indeed turns out that under normal interstellar conditions, except for regions close to shocks and discontinuities, MHD theory essentially allows to conclude a magnetic field behaviour which can be described as a field-freezing into the mass-weighted plasma (ion!) bulk flow with flux-conservation within closed loops, i.e. convected flux tubes, transported with the ion bulk flow $V_{p}$. Under kinetic studies of the behaviour of charge exchanging fluids in the heliosheath regions (see Fahr and Bzowski, 2004a/b; Chashei and Fahr, 2005; Chashei, Fahr and Lay, 2005) it, however, becomes evident that non-negligible deviations from the adopted, highly relaxated HD distribution functions, i.e. shifted Maxwellians, will occur - not only for neutral atoms - but also for ions. These non-equilibrium ion conditions may partly violate the above conclusions and may require a revision of the usual ideal MHD fluid concepts.

Usually, when applying multifluid MHD to the heliosheath problem, one arrives at the following current eqnarray (see

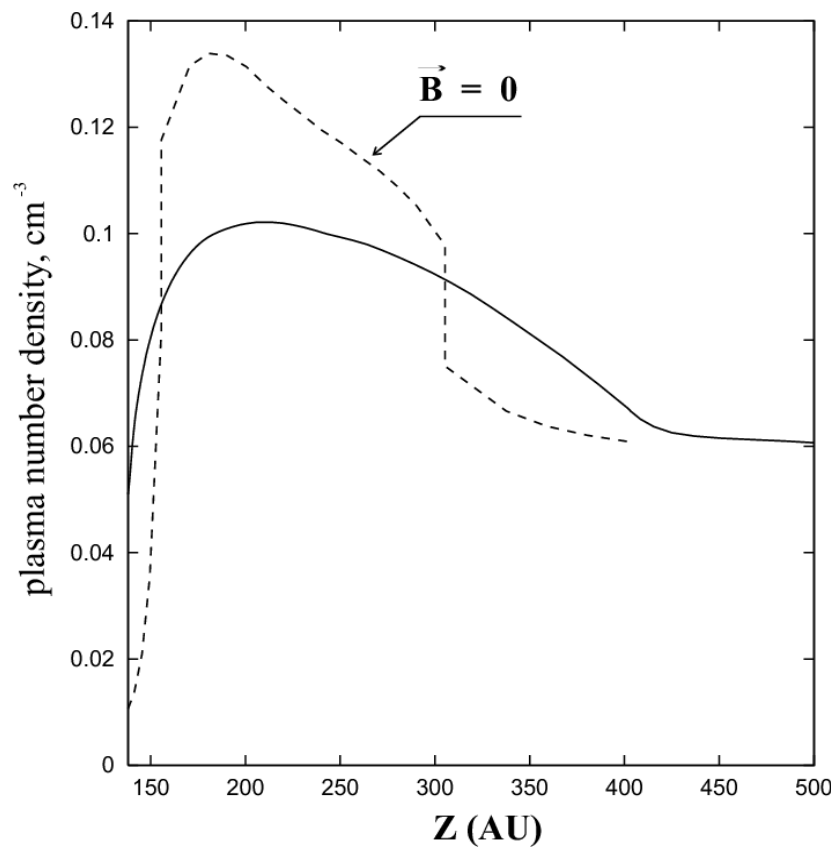

Fig. 3. Solutions for the plasma density as function of the solar distance are shown on the upwind axis: Dashed lines are obtained for vanishing interstellar magnetic field (i.e. $\mathrm{B}=0$ ), fully drawn lines are valid for a finite magnetic field $B=2.5 \mu \mathrm{G} \geq 0$.

e.g. Gombosi, 1998)

$$
\begin{aligned}
\mathbf{j}=\frac{1}{4 \pi} \nabla \times \mathbf{B} & =\frac{\sigma_{0}}{1+\frac{\tau_{e, i}}{\tau_{e, a}}}\left[\frac{1}{e n_{e}} \nabla P_{e}+\left(\mathbf{E}+\mathbf{V}_{p} \times \mathbf{B}\right)\right] \\
& \simeq \sigma_{0}^{*}\left[\left(\mathbf{E}+\mathbf{V}_{p} \times \mathbf{B}\right)\right]
\end{aligned}
$$

where the latter step can only be made under the neglect of electron pressure. Then the above eqnarray can be rewritten in the following form

$\nabla \times\left[\frac{1}{4 \pi \sigma_{0}^{*}} \nabla \times \mathbf{B}\right]=\nabla \times \mathbf{E}+\nabla \times\left[\mathbf{V}_{\mathbf{p}} \times \mathbf{B}\right]$

This eqnarray is even correct, if the electron pressure is kept, since $\nabla \times \nabla P_{e}$ always vanishes. For stationary conditions, i.e. $\nabla \times \mathbf{E}=\partial \mathbf{B} / \partial t=0$, one can try to solve this eqnarray by setting $\mathbf{B}=\mathbf{B}_{\mathbf{0}}+\mathbf{B}_{1}$ with the frozen-in part of the field fulfilling

$\nabla \times\left[\mathbf{V}_{\mathbf{p}} \times \mathbf{B}_{0}\right]=0$

and the diffusive part solving

$\nabla \times\left[\frac{1}{4 \pi \sigma_{0}^{*}} \nabla \times \mathbf{B}\right]=\nabla \times\left[\mathbf{V}_{\mathbf{p}} \times \mathbf{B}_{1}\right]$

The field $\mathbf{B}_{\mathbf{0}}$ is taken to be the completely frozen-in field component convected by the ion bulk flow $\mathbf{V}_{p}$, and the nonfrozen perturbation $\mathbf{B}_{\mathbf{1}}$ is a small non-frozen-in field perturbation due to the kinetic, i.e. non-fluid-like behaviour of the charge-exchanging media. 
Assuming that the remaining, charge-exchange induced electric currents $\mathbf{j}_{e x}=\nabla \times \mathbf{B} / 4 \pi$ are ion currents connected with kinetically induced deviations from the relaxated ion fluid distribution function in connection with charge exchange injected newly created ions, i.e. deviations from the shifted ion Maxwellian, we then can fulfill Eq. (23) by

$\frac{1}{\sigma_{0}^{*}} \mathbf{j}_{e x}=\left[\mathbf{V}_{\mathbf{p}} \times \mathbf{B}_{1}\right]+\nabla \Phi_{B_{1}}$

where for the most general case a scalar field $\Phi_{B_{1}}$ has to be admitted. Now we may aim at the derivation of the charge-exchange induced current $\mathbf{j}_{e x}$ and according to Fahr and Bzowski (2004a) and Chashei, Fahr and Lay (2005) one finds that this current can be given by

$\mathbf{j}_{e x} \simeq e \delta n \cdot\left(\left(\mathbf{V}_{a}-\mathbf{V}_{p}\right) \cdot \mathbf{B}_{\mathbf{0}} / B_{0}\right)\left(\mathbf{B}_{\mathbf{0}} / B_{0}\right)$

where $\delta n$ is the partial ion density of local perturbations induced by freshly charge-exchange injected, non-relaxated ions, establishing and maintaining a nonequilibrium perturbation $\delta n$ which, for stationary cases, is given by the balance condition between injection and removal of new ions in the form (see Fahr and Bzowski, 2004b; Chashei, Fahr and Lay, 2005)

$\delta n \simeq n_{a} n_{p} V_{\mathrm{rel}}(H, p) \sigma_{\mathrm{rel}}(H, p) \tau_{p p}$

where $\mathrm{H}$-atom densities and unperturbed proton densities are denoted by $n_{a}$ and $n_{p}$, respectively, where $\sigma_{\text {rel }}$ is the average charge exchange cross section of proton - $\mathrm{H}$-atom collisions with an average relative velocity of $V_{\text {rel }}(H, p)$ given by:

$V_{\text {rel }}(H, p)=\sqrt{\frac{128}{9 \pi m_{p}}\left(T_{H}+T_{p}\right)+\left(\mathbf{V}_{a}-\mathbf{V}_{p}\right)^{2}}$

Furthermore $\tau_{p p}$ is the period of the most effective ion (proton) relaxation process, i.e. either Coulomb relaxation or wave-particle relaxation (see Chashei, Fahr and Lay, 2005). Then with the assumption $\Phi_{B_{1}}=$ const one obtains with Eqs. (25), (26) and (27) the following eqnarray for a particular solution for $\mathbf{B}_{1}$

$\frac{1}{\sigma_{0}^{*}} \Gamma\left(\left(\mathbf{V}_{a}-\mathbf{V}_{p}\right) \cdot \frac{\mathbf{B}_{\mathbf{0}}}{B_{0}}\right)\left(\frac{\mathbf{B}_{\mathbf{0}}}{B_{0}}\right)=\left[\mathbf{V}_{p} \times \mathbf{B}_{1}\right]$

where $\Gamma=\Gamma(H, p)=e n_{a} n_{p} V_{\text {rel }}(H, p) \sigma_{\text {rel }}(H, p) \tau_{p p}$.

To solve for $\mathbf{B}_{1}$ we carry out a vector multiplication of the above eqnarray with $\mathbf{V}_{p}$ and obtain

$$
\begin{aligned}
& \frac{1}{\sigma_{0}^{*}} \Gamma \frac{\left(\mathbf{V}_{a}-\mathbf{V}_{p}\right) \cdot \mathbf{B}_{\mathbf{0}}}{B_{0}^{2}}[ {\left[\mathbf{V}_{p} \times \mathbf{B}_{\mathbf{0}}\right] } \\
&=\frac{1}{c^{2}}\left[\mathbf{V}_{p}\left(\mathbf{V}_{p} \cdot \mathbf{B}_{1}\right)-\mathbf{B}_{1} V_{p}^{2}\right]
\end{aligned}
$$

Since the left side only contains a vector perpendicular to $\mathbf{V}_{p}$ one thus finds that $\mathbf{B}_{1}$ also has to be perpendicular to $\mathbf{V}_{p}$, and hence that the following relation should be valid:

$\mathbf{B}_{1}=-\frac{1}{\sigma_{0}^{*}} \Gamma \frac{c\left(\mathbf{V}_{a}-\mathbf{V}_{p}\right) \cdot \mathbf{B}_{\mathbf{0}}}{V_{p}^{2} B_{0}^{2}}\left[\mathbf{V}_{p} \times \mathbf{B}_{\mathbf{0}}\right]$

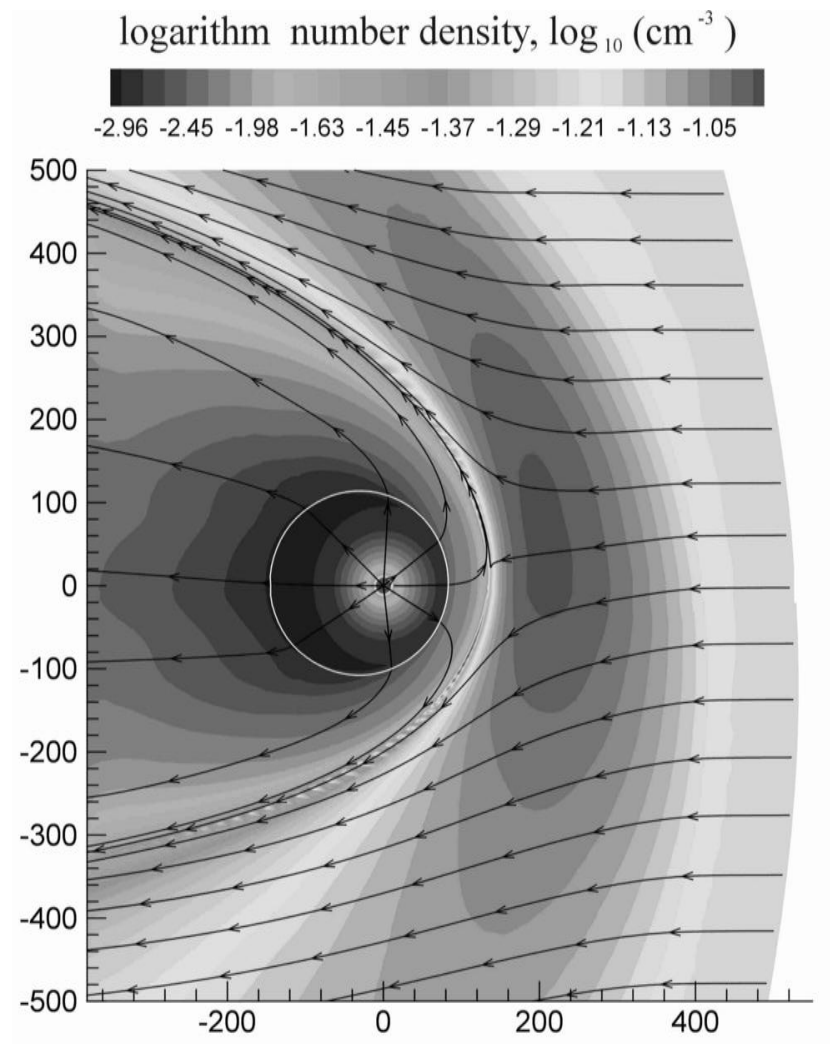

Fig. 4. Plasma density contours and plasma flow lines are plotted for the case of a finite interstellar magnetic field $B=2.5 \mu G \geq 0$.

which means that we simply can calculate a particular solution for $\mathbf{B}_{\mathbf{1}}$ from first order MHD quantities like: $n, T_{p}, \mathbf{V}_{p}$ and $\mathbf{B}_{0}$ given within the frame of a multi-fluid MHD simulation which models the outer heliospheric interface.

To evaluate on the order of magnitude of the kineticallyinduced additional fields $B_{1}$ one may write for regions, where $\left|\mathbf{V}_{a}\right| \simeq\left|\mathbf{V}_{p}\right|$ is valid, and with representative data for the outer heliosheath like:

$$
\begin{aligned}
& n_{a}=0.1 \mathrm{~cm}^{-3} \\
& n=0.1 \mathrm{~cm}^{-3} \\
& V_{\text {rel }}(H, p) \simeq\left|\mathbf{u}_{a}\right| \simeq 20 \mathrm{~km} / \mathrm{s} \\
& \sigma_{\text {rel }}(H, p) \simeq 4 \cdot 10^{-15} \mathrm{~cm}^{2} \\
& \tau_{p p} \simeq 4 \cdot 10^{7} \mathrm{~s} \\
& \sigma_{0}^{*} \simeq n e^{2} \tau_{e, i} / m_{e} \\
& \tau_{e, i} \simeq 2.8 \cdot 10^{-1} \frac{T_{e} \sqrt{T_{e}}}{n L_{C}} \simeq 11.2 \cdot 10^{5} \mathrm{~s} \\
& \omega_{g}=\frac{e B_{0}}{m_{e} c} \simeq 18.0 \mathrm{~s}^{-1} \\
& \left(L_{C}=\text { Coulomb logarithm }\right)
\end{aligned}
$$


the following relation:

$\frac{\left|\mathbf{B}_{1}\right|}{\left|\mathbf{B}_{0}\right|} \simeq \frac{1}{\sigma_{0}^{*}} \Gamma c \frac{1}{\left|\mathbf{B}_{0}\right|}=\left(\tau_{p p} / \tau_{e, i}\right) \frac{v_{a}}{\omega_{g}} \simeq 10^{-4}$

with the charge exchange

frequency $v_{a}=n_{a} V_{\text {rel }}(H, p) \sigma_{\text {rel }}(H, p) \simeq 3 \cdot 10^{-8} \mathrm{~s}^{-1}$. Only for regions close to the inner and outer surface of the front part of the heliopause where $\left|\mathbf{V}_{a}\right| \gg\left|\mathbf{V}_{p}\right|$ is valid, one may find results different from the above relation, namely rather given by:

$$
\frac{\left|\mathbf{B}_{1}\right|}{\left|\mathbf{B}_{0}\right|} \simeq\left(\tau_{p p} / \tau_{e, i}\right) \frac{v_{a}}{\omega_{g}} \frac{\left|\mathbf{V}_{a}\right|}{\left|\mathbf{V}_{p}\right|} \simeq 10^{-4} \frac{\left|\mathbf{V}_{a}\right|}{\left|\mathbf{V}_{p}\right|}
$$

\section{Conclusions}

As we have shown in the sections above, ideal MHD conditions for motions of partially ionized and magnetized plasmas, usually leading to magnetic fields exactly frozen into the ion fluid, may break down under the influence of chargeexchange induced electric currents. This we have shown when deriving the generalized form of the induction eqnarray for partially ionized gases with low or moderate ionization degrees $s$ where charge exchange coupling between $\mathrm{H}$ atoms and protons becomes magnetohydrodynamically important (i.e. ionization degrees $s \geq 0.5$ ). The additional electric currents that are induced lead to a term in this induction eqnarray which describes magnetic field diffusion, a kind of freedom of the magnetic fields with respect to a strict comotion with the ion fluid. This term becomes especially important near structures where abrupt spatial changes of the magnetic field in magnitude or direction occur, i.e. for instance as expected near the heliopause or the outer heliospheric bowshock. In the following we shall give a short estimate of how much influence the charge-exchange induced non-ideality has on the transition profile of the interstellar magnetic field at the bowshock.

In the following we intend to briefly estimate the chargeexchange induced non-idealness at the MHD bowshock transition. Assuming the situation that the LISM magnetic field is oriented perpendicular to the LISM inflow onto the heliosheath, one then would obtain with the extended induction eqnarray given by Eq. (12) the following relation describing the behaviour of $\boldsymbol{B}$ and $\boldsymbol{V}$ at the passage of the magnetized LISM plasma over the bowshock:

$\nabla \times(\boldsymbol{V} \times \boldsymbol{B})=\kappa \Delta \boldsymbol{B}$

Applying this to the region near the symmetry axis of the LISM inflow (i.e. the so-called $z$-axis) one can then, due to symmetry reasons (i.e. $\partial / \partial x=\partial / \partial y=0$ ), derive from the above vector relation the following scalar differential eqnarray:

$-\frac{d}{d z}\left(V_{z} B_{x}\right)=\kappa \frac{d^{2} B_{x}}{d z^{2}}$
We shall solve this differential eqnarray explicitly in a forthcoming paper, since the detailed elaboration of this solution via reducing the above eqnarray to a Ricatti-type differential eqnarray would extend this paper here too much. Instead we give an estimate of the solution by entering into a dimensional argumentation. Let us assume a transition scale $\Delta$ to be typical for the bowshock transition. Then, on the basis of purely dimensional arguments, one can simplify the above eqnarray to the form:

$\frac{V_{z 1} B_{1 x}-V_{z 2} B_{2}}{\Delta} \simeq \kappa \frac{B_{2 x}-B_{1 x}}{\Delta^{2}}$

leading to the following result:

$B_{2 x} \simeq B_{1 x} \frac{V_{1 z}+(\kappa / \Delta)}{V_{2 z}+(\kappa / \Delta)}=B_{1 x} \frac{V_{1 z}}{V_{2 z}} \frac{1+K_{1}}{1+K_{2}}$

What one notices in the above expression is that for $\kappa=0$ one regains the normal frozen-in flux behaviour valid for ideal MHD, for $\kappa \geq 0$ one, however, obtains a non-ideal behaviour, i.e. violation of magnetic flux conservation, described by

$B_{1 x} \leq B_{2 x} \leq B_{1 x} \frac{V_{1 z}}{V_{2 z}}$

Taking the values already used earlier in this paper for the quantity $\kappa=(1-s)^{2} v_{A}^{2} \tau_{p H}$ one would find with $L \simeq 50 \mathrm{AU}$ :

$K_{1}=\left(\kappa / V_{1 z} \Delta\right) \simeq\left(\frac{\kappa}{L V_{1 z}}\right)\left(\frac{L}{\Delta}\right)=\left(\frac{L}{\Delta}\right)$

and:

$K_{2}=\left(\kappa / V_{2 z} \Delta\right) \simeq\left(\frac{\kappa}{L V_{1 z}}\right)\left(\frac{V_{1 z}}{V_{2 z}}\right)\left(\frac{L}{\Delta}\right)=\sigma\left(\frac{L}{\Delta}\right)$

where $\sigma=V_{1 z} / V_{2 z}$ denotes the compression ratio at the bowshock. Taking the value for the most efficient proton relaxation period $\tau_{e p} \simeq 2.5 \cdot 10^{7} \mathrm{~s}$ calculated by Chashei, Fahr and Lay (2005) one may then estimate the shock transition scale by $\Delta \simeq V_{1 z} \tau_{e p}=4 \mathrm{AU}$ and finds

$B_{2 x} \simeq B_{1 x} \sigma \frac{1+(L / \Delta)}{1+\sigma(L / \Delta)} \simeq B_{1 x} \sigma \frac{13.5}{1+\sigma 12.5}$

meaning that the magnetic field compression to be expected under these non-ideal conditions is much less pronounced compared to the ideal MHD case with $B_{2 x}=B_{1 x} \sigma$.

Acknowledgements. This work was supported by INTAS (grant no. 2001-0270), the Russion Foundation for Basic Research (project nos. 03-02-04020, 04-01-00594, 04-02-16559), DFG (grant np. 436 RUS 113/110/12-3), and the Basic Research Program of the Russian Academy of Sciences. We are grateful to the referees for helpful remarks and comments.

Edited by: K. Scherer

Reviewed by: two referees 


\section{References}

Alexashov, D. B., Baranov, V. B., Barskii, E. V., and Myasnikov, A. V.: Axially symmetric MHD model for the solar wind - LISM interaction, Astron. Lett., 26, 743-749, 2000.

Baranov, V. B., Krasnobaev, K. V., and Kulikovskii, A. G.: On the model of the solar wind - interstellar medium interaction with two shocks, Dokl. Akad. Nauk USSR, 194, 41-44, 1970.

Baranov, V. B. and Malama, Yu. G.: Model of the solar wind interaction with the local interstellar medium - Numerical solution of self-consistent problem, J. Geophys. Res., 98, 15 157-15 163, 1993.

Baranov, V. B. and Fahr, H.-J.: On nonideal MHD properties of the partially ionized interstellar gas, J. Geophys. Res., 108, SSH 4-1, CiteID 1110, DOI: 10.1029/2001JA009221, $2003 \mathrm{a}$.

Baranov, V. B. and Fahr, H.-J.: Reply to comment by V. Florinski and G. P. Zank on "On nonideal MHD properties of the partially ionized interstellar gas" , J. Geophys. Res., SSH 5-1, CiteID 1439, DOI: 10.1029/2003JA010118, 2003 b.

Chashei, I. V. and Fahr, H.-.J.: Ion relaxation processes in the heliospheric interface: how perturbed are ion distribution functions?, Adv. Space Res., 35, 2078-2083, 2005.

Chashei, I. V., Fahr, H.-J., and Lay, G.: Non-Equilibrium Distribution Functions in the Heliospheric Interface and Their Relaxation by Local Wave Particle Interactions, Sol. Phys., 226, 163-185, 2005.

Cowling, T. G.: Magnetohydrodynamics, Adam Hilger, London, U.K., 1976.
Fahr, H.-J., Kausch, T., and Scherer, H.: A 5-fluid hydrodynamic approach to model the solar system-interstellar medium interaction, Astron. Astrophys., 357, 268-283, 2000.

Fahr, H.-J. and Bzowski, M.: A kinetic control of the heliospheric interface hydrodynamics of charge-exchanging fluids, Astron. Astrophys., 424, 263-276, 2004a.

Fahr, H.-J. and Bzowski, M.: Charge-exchange-induced perturbations of ion and atom distribution functions in the heliospheric interface, AIP Conf. Proc. 719: Physics of the Outer Heliosphere 719, 373-380, 2004b.

Florinskii, V. and Zank, J.: Comment on "On nonideal MHD properties of the partially ionized interstellar gas" by V. B. Baranov and H. J. Fahr, J. Geophys. Res., 108, SSH4-1, 1438-1439, DOI 10.1029/2003JA009950, 2003.

Florinskii, V., Zank, G. P., and Pogorelov, N. V.: Galactic cosmic ray transport in the global heliosphere, J. Geophys. Res., 108, SSH1-1, 1228-1235, DOI 10.1029/2002JA009695, 2003.

Gombosi, T. I.: In "Physics of the space environment" Cambridge Atmospheric and Space Sciences Series, edited by Houghton, J. T., Rycroft, M. J., and Dessler, A. J., Cambridge University Press, Michigan, 72, formula 4.75 et seqq, 1998.

Izmodenov, V. V., Alexashov, D. B., and Myasnikov, A. V.: Direction of the interstellar $\mathrm{H}$ atom inflow in the heliosphere: Role of the interstellar magnetic field, Astron. Astrophys., 437, L35L38, 2005.

Kulikovskii, A. G. and Lyubimov, G. A.: Magnetohydrodynamics, Addison-Wesley-Longman, Reading, Mass., 1965. 